Research Article

\title{
Diagnostic Value of Chest CT Images Based on Full Model Iterative Reconstruction Algorithm for Lung Cancer Patients
}

\author{
Xia Li $\mathbb{D},{ }^{1}$ Zhanqiang Song $\mathbb{D}^{1},{ }^{1}$ Guiping Shen $\mathbb{D}^{2},{ }^{2}$ Ying Huang $\mathbb{D},{ }^{3}$ and Junyu Chen $\mathbb{D}^{1}$ \\ ${ }^{1}$ Department of Radiology, \\ Wuhan Fourth Hospital (Puai Hospital, Tongji Medical College, Huazhong University of Science and Technology), \\ Wuhan 430000, China \\ ${ }^{2}$ Department of Radiology, General Hospital of the Central Theater Command of the PLA, Wuhan 430000, China \\ ${ }^{3}$ Pulmonary and Critical Care Medicine, Wuhan Hospital of Traditional Chinese and Western Medicine, Wuhan, China
}

Correspondence should be addressed to Zhanqiang Song; 1731190886@xzyz.edu.cn

Received 19 June 2021; Accepted 11 August 2021; Published 10 September 2021

Academic Editor: Gustavo Ramirez

Copyright (C) 2021 Xia Li et al. This is an open access article distributed under the Creative Commons Attribution License, which permits unrestricted use, distribution, and reproduction in any medium, provided the original work is properly cited.

\begin{abstract}
Objective. To evaluate the value of low-dose CT scanning and full model iteration recombinant technology peripheral lung cancer in the paper using whole model iterative reconstruction algorithm and compare iterative model-wide restructuring, reorganization part of an iterative algorithm, affecting filtered back projection image quality. Method. Fifty-two patients with peripheral lung cancer, all of whom were diagnosed by pathological biopsy, were selected for the study. All patients received three scans of low-dose chest CT, next-low-dose, and low-dose, after which the raw data of three different doses were reconstructed using filtered back-projection, iterative partial algorithm reconstitution, and reconstructed full-model iteration, respectively, and the effect of each algorithm on the processing of chest CT images of peripheral lung cancer at different doses and the diagnosis of the disease were compared after the reconstitution was completed. Results. The average effective radiation dose for the low-dose group was $(0.3 \pm 0.02) \mathrm{mSv}$ At each dose level, image noise objective recombinant whole iterative model $<$ part of the reorganization of the iterative algorithm $<$ filtered back projection, the difference was significant. In the case of lung lesions, the full-model iterative algorithm has similar evaluation power to the LD-partial iterative algorithm. When a patient's body mass index $(\mathrm{BMI})>25 \mathrm{~kg} / \mathrm{m}^{2}$, the whole model iteration reorganization image quality is reduced, but the lesions-to-noise ratio (SNR) is unaffected. Conclusion. The combination of a very low dose of recombinant iterative model as compared to full-dose low-dose chest CT dose can be reduced to $88 \%$ but does not reduce the overall image quality and can show good radiological signs of peripheral lung cancer and not affect BMI patients.
\end{abstract}

\section{Introduction}

According to the latest cancer statistics, lung cancer has the highest incidence and cancer mortality. Over the past decade, new cases and deaths in people with lung cancer are also high. Currently, low-dose CT is an effective method widely used for early diagnosis and monitoring of treatment of lung cancer, and based on the full iterative CT recombinant technology iterative model reorganization, it can significantly reduce the radiation dose and improve image signal-to-noise ratio (SNR) [1]. At present, domestic and foreign rare-wide restructuring used in iterative model of peripheral lung cancer is reported in the literature. In summary, this study hopes to use the fullmodel iterative algorithm for low-dose chest CT diagnosis of peripheral lung cancer, and to verify the value of using the full-model iterative algorithm for clinical diagnosis of chest CT of peripheral lung cancer by evaluating the image quality, noise profile, and lung cancer lesion identification of each algorithm at different doses of chest CT [1].

\section{Materials and Methods}

2.1. Clinical Data. Patients who were diagnosed with peripheral lung cancer in our hospital from March 2017 to May 2019 were considered. Inclusion criteria: (1) patients who were diagnosed with peripheral lung cancer by biopsy and imaging for CT reexamination; (2) aged $\geq 18$ years; (3) able to hold their breath for at least 10 seconds at a time. Exclusion 
criteria: (1) patients who are pregnant or lactating; (2) patients with poor general condition are not suitable for CT examination; (3) CT image artifacts make it impossible to observe the lesion. Finally, 52 patients were selected, including 24 males and 28 females, aged $41-86$ years, with an average of $58.4 \pm 10.9$ years.

2.2. Inspection Method. In this study, three CT scans were performed in all patients, namely, low-dose chest CT, the next-lower-dose CT, and low-dose three CT scans, with an interval of 1 week between the two low-dose scans. The CT images obtained from the three scans were processed using the filtered back-projection algorithm, the partial iterative recombination algorithm, and the full-model iterative recombination algorithm, and finally nine CT images were obtained for each patient, i.e., a low-dosefiltered back projection/recombinant iterative algorithm part/whole recombination model iteration, the next lower dose-filtered back projection/recombination portion iterative algorithm/recombinant full model, and iterative filtered back projection low dose/recombination portion iterative algorithm/recombinant full model iteration.

2.3. Radiation Dose. The dose length product (DLP), CT dose index volume (CTDIvol), and effective radiation dose (ED) of each patient's three scans were recorded; the calculation formula is $\mathrm{ED}=\mathrm{DLP} \times 0.014$.

2.4. Evaluation of Image Quality. Objective evaluation index: a radiologist measures the image noise (SD) and CT value and calculates SNR lesions of each group; specific method: place the region of interest (ROI) $15-20 \mathrm{~mm}^{2}$ in the trachea above the tracheal bifurcation and measure the image SD; place ROI20 30 $\mathrm{mm}^{2}$ in the descending aorta at the tracheal bifurcation to measure CT value. As far as possible, select the level that shows the largest extent of the lesion, avoid the liquid necrosis area and the larger trachea, and place the ROI and measure its CT value (CTa), and at the same level, select the subcutaneous fat tissue of the chest and place the ROI and measure its SD as the background noise (SDa). SNR lesion calculation formula: $\mathrm{SNR}$ lesion $=\mathrm{CTa} / \mathrm{SDa}$. To ensure the reliability of the results, the above measurement is repeated three times, and the average value is taken as the final result [2].

Image overall subjective evaluation indicators: blind and randomized principles are used to evaluate images by two senior physicians. When the two opinions are different, the agreement is reached through consultation; the overall image subjective evaluation content: image graininess, image artifacts, and normal anatomy structure (pulmonary fissure, bronchial wall below the subsegment, blood vessel within $1 \mathrm{~cm}$ below the pleura, and pericardium) show clarity. A 4level score is used: 1 point (poor), image noise and artifacts are serious, the structure display is fuzzy, and diagnostic information cannot be obtained; 2 points (medium), the image has noise and artifacts, and the structure display is general, barely to obtain diagnostic information; 3 points (good), the image noise and artifacts are lighter, the structure is displayed clearly, and effective diagnostic information can be obtained; 4 points (very good), the image noise or artifacts are less, and the structure display is very clear enough to obtain effective diagnostic information.

Lesion display evaluation index: Since LD-HIR is mainly used clinically as a subjective evaluation index for lesion diagnosis, this study used to compare the lesion display of three different reconstructed algorithm images with LD-HIR images under SLD-CT and ULD-CT to evaluate the application value of the algorithm. The characteristics of the lesions evaluated included calcification, necrosis, location, burr sign, pleural depression sign, vacuole, and ground glass shadow. Use a grade 2 score: 1, absent; 2 , exists. Lesion size: measure the maximum transverse diameter at the largest level of the lesion, repeat the measurement three times each time, and take the average; finally, to judge the nature of the lesion, use a grade 2 score: 1 , benign; 2 , malignant.

2.5. Image Super-Resolution Reconstruction. The model is described as follows: with $p m \times n$ LR observation image $\left\{y_{k}\right\}_{k}^{p}=1$, according to the image degradation model, there are

$$
y_{k}=D B_{k} z+n_{k}, \quad 1 \leq k \leq p .
$$

In the model of (1), $y_{k}$ is the $N \times 1(N=m n)$ vector composed of the $k m \times n$ LLR observation $y_{k}$ images after dictionary sorting, if $r_{1}$ and $r_{2}$ are the down sampling factors in the horizontal and vertical directions, respectively; then, $Z$ is the $\mathrm{HH}$ image of $r_{1} m \times r_{2} n z$-a vector of $r_{1} r_{2} N \times 1$ formed by dictionary sorting, $M_{k}$ is the affine transformation matrix of $r_{1} r_{2} N \times r_{1} r_{2} N, B_{k}$ is the fuzzy matrix of $r_{1} r_{2} N \times r_{1} r_{2} N, D$ is $N \times r_{1} r_{2} N$, the down sampling matrix, and $n_{k}$ is the Gaussian white noise of $N \times 1$ vector. It can be obtained from model (1) that image SSR reconstruction needs to solve the following minimization problem:

$$
\min J(z): J(z)=\sum_{k}\left\|y_{k}-D B_{k} M_{k} z\right\|^{2} .
$$

It must be adjusted by adding a balance term.

$$
\min J(z): J(z)=\sum_{k}\left\|y_{k}-D B_{k} M_{k} z\right\|^{2}+\lambda_{I}(z) \text {. }
$$

In equation (3), $\lambda_{I}$ is the balance factor, $\rho(z)$ is the regularization term, and there are many ways to determine $\rho(z)$; let

$$
\rho_{\mathrm{BTV}}(z)=\sum_{l=-w}^{w} \sum_{m=0}^{w} a^{|m|+|l|}\left\|z-S_{x}^{l} S_{y}^{m} z\right\|^{2},
$$

where $S_{x}^{l}$ and $S_{y}^{m}$ in equation (4) are the shift matrices that shift the image $z$ by $l$ and $m$ pixels in the horizontal and vertical directions, respectively, and $\alpha(0<\alpha<1)$ is the weighting coefficient.

In summary, a single constant $\alpha$ will have a double impact on the reconstructed HR image, so how to choose the 
appropriate $\alpha$ is very important for the quality of the final reconstructed HR image. This article introduces an adaptive weighting coefficient $\alpha_{i}$, according to the specific difference between the edges, and the smooth part of the image can not only sharpen the edge of the image but also smooth the rest of the image and suppress noise, thereby effectively ensuring the image quality. The specific methods are as follows [3]. (4):

Suppose $z^{\prime}(l, m)=S_{x}^{l} S_{y}^{m} z$ can be obtained from formula

$$
\rho_{\mathrm{BTV}}(z)=\sum_{l=-w}^{w} \sum_{m=0}^{w} a^{|m|+|l|}\left\|z-z^{\prime}(l, m)\right\|^{2} .
$$

Let $z-z^{\prime}(l, m)=\left[z_{1}(l, m), z_{2}(l, m), \ldots, z_{r_{1} m \times r_{2} n}(l, m)\right]$ have the physical meaning: element $z_{i}(l, m)$ in vector $z-z^{\prime}(l, m)$ is the difference between the pixels at the same position in the reconstructed image $z$ and its surrounding pixels. In the gray-scale image, $0 \leq z_{i}(l \cdot m) \leq 255$; if $z_{i}$ is too large, it means that the reconstructed image $z$ has a mutation around the $i$ th pixel, i.e., there is a sudden change around the $i$ pixel, that is, there is an edge around the $i$ point, so we hope that the reconstruction algorithm can sharpen the edge around the $i$ point to highlight the display effect of the edge; otherwise, it, $z_{i}(l . m)$, is too small, which means that the $i$-th pixel in the image is smooth, and we hope that the reconstruction algorithm can suppress noise. In summary, in order to achieve the purpose of SSR reconstruction according to the characteristics of different images, the adaptive bilateral full variation regularization term $\rho_{\mathrm{SBTV}}(z)$ is introduced as follows:

$$
\rho_{\mathrm{BTV}}(z)=\sum_{l=-w}^{w} \sum_{m=0}^{w}\left\{\sum_{i=1}^{r_{1} m \times r_{2} n}\left(a_{i}^{|m|+|l|} z_{i}(l, m)\right)^{2},\right.
$$

where $a_{i}$ in equation (6) is an adaptive weighting coefficient, which changes according to the difference of each pixel of the reconstructed HR image, which fully guarantees the adaptability of the algorithm, thereby theoretically improving the quality of the reconstructed HR image.

\section{Results}

3.1. Radiation Dose. The DLP, CTDIvol, and ED results of the three scanning schemes are shown in Table 1. Compared with the LD-CT group, the ED of the SLD-CT group and ULD-CT decreased by $68 \%$ and $88 \%$, respectively. For the ULD-CT group, the ED was as low as $0.3 \pm 0.02 \mathrm{mSv}$.

3.2. Image Quality. Objective evaluation: objective video quality evaluation results are shown in Table 2. As can be seen from Figure 1, the SD values of CT images for three different degrees of model iteration algorithms show an overall decreasing trend $(F=242.8, P<0.05)$. SNR value lesions were in each group, increased tendency scanning group $(F=99.7$, $P<0.05)$. Low dose-part of the reorganization of the iterative algorithm, the second lowest dose-iterative pairwise comparison between model-wide restructuring, reorganization of the whole model iteration, no statistically significant $(P>0.05)$ image SD value differences. Recombinant full iterative model, the image SD under the influence BMI (Table 3), and the image
SD BMI $\geqq 25$ were significantly higher than $\mathrm{BMI}<25$ group $(t=3.8, P<0.001)$, while no lesions in both groups SNR in BMI were significantly different $(t=0.75, P>0.05)$. There was no significant $(P>0.05)$ (Figure 2) CT value difference between the 9 measured image groups.

Subjective evaluation entire image: the image overall subjective evaluation results are shown in Table 4 and Figures 3-5. The results showed that in the CT images obtained by different algorithms in the LD-CT group, the SLD-CTgroup, and the ULD-CT group, the subjective evaluation scores of the overall image quality processed by the HIR algorithm and the IMR algorithm were higher than those in the FBP group, but the differences were not significant and not statistically significant $\left(\chi^{2}=0.3, P>0.05\right)$. The subjective evaluation scores of FBP and HIR algorithm-processed images in the ULD-CT group were significantly lower than those in the SLD-CTgroup, and the difference was statistically significant $\left(\chi^{2}=9.2\right.$, $P<0.05)$ score. Low-dose part of the reorganization of the iterative algorithm and the next lower dose iterative modelwide restructuring had no statistically significant $\left(\chi^{2}=1.6\right.$, $P>0.05)$ difference between the full model iterations restructuring group score. Iterative recombinant full model, $\mathrm{BMI} \leq 25$ group score, is above $\mathrm{BMI}>25$ group $(t=3.5$, $P<0.05$ ) (Table 4).

Subjective evaluation indicators of lesions: the signs of peripheral lung cancer, necrosis, location, burr sign, pleural depression sign, vacuole, and ground glass shadow showed the results in the three groups of LD-HIR, ULD-FBP, ULD-HIR, and ULD-IMR. There was no statistical difference. ULD-FBP showed a significant difference in calcification compared with the other three groups $\left(\chi^{2}=9.2, P<0.05\right)$. The false positive rate of ULD-FBP in the evaluation of calcification was $14 \%$. In measuring the size of the lesion, there was no statistical difference between the four groups $(P>0.05)$. The size of the lesion measured by LD-CT ranged from 1.09 to $4.55 \mathrm{~cm}$, with an average value of $2.11 \pm 1.3 \mathrm{~cm}$. The qualitative diagnosis of the lesion was consistent among the groups. The results were 3 cases benign and 49 malignant [4].

\section{Discussion}

This study evaluated the use of tube voltage $(80 \mathrm{kVp})$ and tube current $(20 \mathrm{mAs})$ to obtain the feasibility of a very low dose and displays peripheral lung cancer by using the latest full model iterative algorithm restructuring. In this study, a recombinant full model iteration low-dose group was $0.3 \pm 0.02 \mathrm{mSv}$; the low-dose group was compared to $88 \%$ reduction of radiation dose, without reducing image quality. Full model using iterative techniques of recombinant CT system model and a statistical model is corrected, taking into account not only the statistical noise of the nature of the data but also the optical properties of the detection system, thereby improving image quality. When studies have proposed the model of iterative recombinant techniques (MBIR) based on the combination, ED low $(0.16 \pm 0.006 \mathrm{mSv})$, the ability to show subtle anatomy of the normal lung and lung detecting small nodules remains high; studies have also reported when using recombinant techniques, iterative full model can be reduced to $69 \%$ 
TABle 1: Comparison of the radiation dose of three groups of different dose scans.

\begin{tabular}{lccc}
\hline Group & CTDIvol $(\mathrm{mGy})$ & DLP $(\mathrm{mGy} \cdot \mathrm{cm})$ & ED $(\mathrm{mSv})$ \\
\hline LD-CT & $4.1 \pm 0.8$ & $160.1 \pm 35.0$ & $2.2 \pm 0.5$ \\
SLD-CT & $1.3 \pm 0.3$ & $51.3 \pm 12.3$ & $0.7 \pm 0.2$ \\
ULD-CT & $0.5 \pm 0$ & $19.6 \pm 1.7$ & $0.3 \pm 0.02$ \\
$F$ value & 745.9 & 615.7 & 615.7 \\
$P$ value & $<0.001$ & $<0.001$ & $<0.001$ \\
\hline
\end{tabular}

TABle 2: Comparison of CT value, SD, and SNR lesions in each group.

\begin{tabular}{|c|c|c|c|}
\hline Parameter & FBP & HIR & IMR \\
\hline & & $L D-C T$ & \\
\hline CT value (HU) & $40.2 \pm 8.5$ & $41.5 \pm 7.9$ & $40.6 \pm 8.0$ \\
\hline $\mathrm{SD}(\mathrm{HU})$ & $25.1 \pm 4.1$ & $17.1 \pm 2.3$ & $10.2 \pm 1.1$ \\
\hline \multirow[t]{2}{*}{ SNR lesions } & $2.0 \pm 0.7$ & $2.5 \pm 0.9$ & $4.3 \pm 1.5$ \\
\hline & & $S L D-C T$ & \\
\hline CT value (HU) & $43.9 \pm 8.4$ & $42.9 \pm 8.5$ & $42.0 \pm 7.1$ \\
\hline $\mathrm{SD}(\mathrm{HU})$ & $51.2 \pm 9.9$ & $33.7 \pm 5.6$ & $15.2 \pm 1.7$ \\
\hline \multirow[t]{2}{*}{ SNR lesions } & $1.1 \pm 0.4$ & $1.5 \pm 0.5$ & $3.3 \pm 1.1$ \\
\hline & & $U L D-C T$ & \\
\hline CT value (HU) & $49.0 \pm 12.5$ & $45.8 \pm 8.5$ & $43.8 \pm 7.1$ \\
\hline SD (HU) & $75.0 \pm 24.01$ & $47.3 \pm 12$ & $18.3 \pm 4.6$ \\
\hline SNR lesions & $0.8 \pm 0.4$ & $1.2 \pm 0.4$ & $2.9 \pm 0.9$ \\
\hline
\end{tabular}
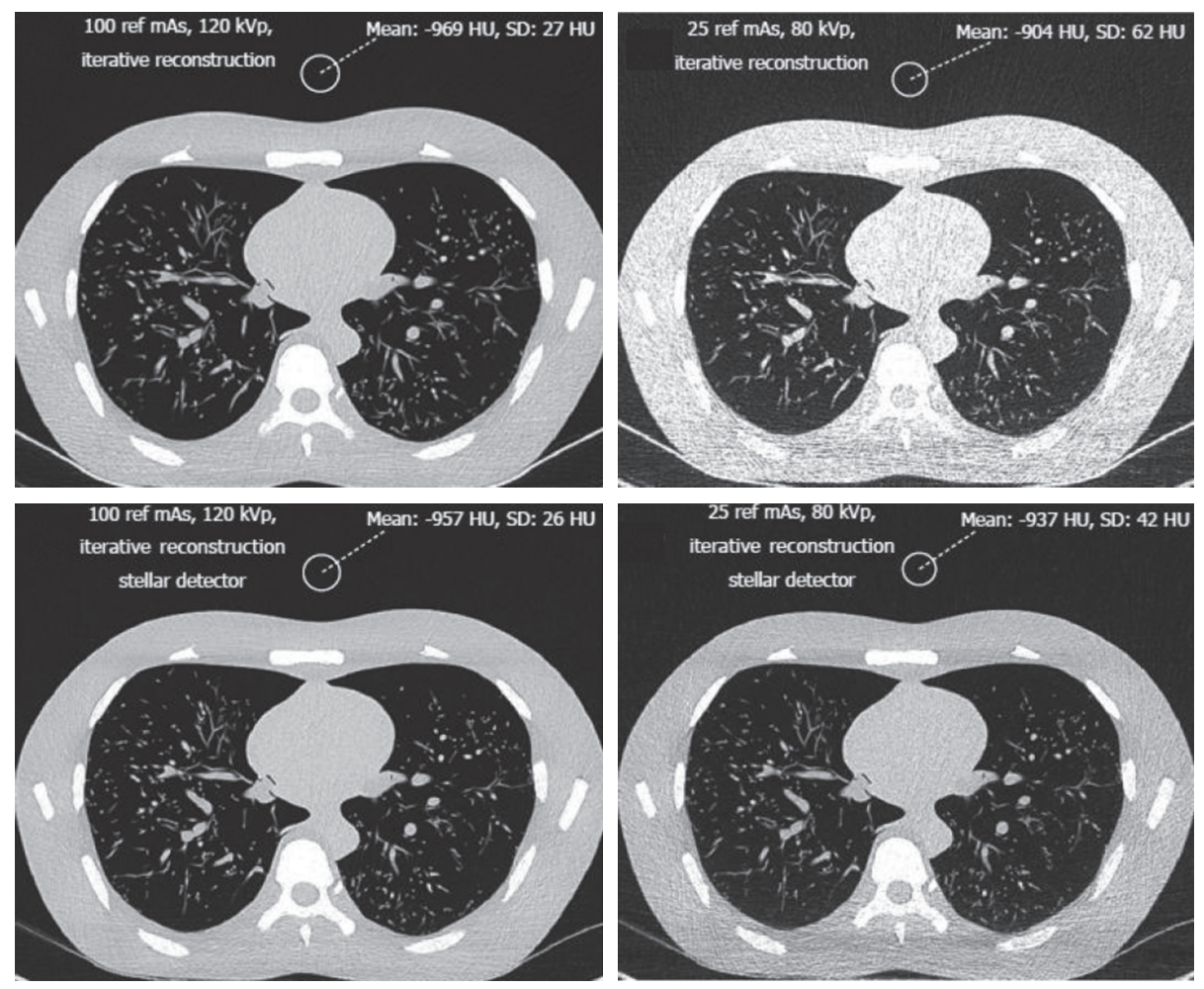

FIGURE 1: Comparison of noise between different doses and different algorithms.

$(0.9 \pm 0.03 \mathrm{mSv})$ dose. The full-model iterative algorithm was better at assessing the sharpness of lung nodule margins than the conventional filtered inverse projection dose and lowdose recombination iterative algorithms; ultra-low-dose full model iterative recombinant SD values were $18.3 \pm 4.6 \mathrm{HU}$.
There was no significant difference in SD values, SNR, and low-dose lesion fraction for low-dose CT images.

The subjective evaluation results showed that the dose in the full model iterative recombination group was as low as $0.3 \pm 0.02 \mathrm{mSv}$, and the normal anatomical structure and lesion 
TABLE 3: Comparison of SD, SNR lesions, and overall image quality scores of different levels of dose, different BMI in IMR algorithm.

\begin{tabular}{|c|c|c|c|}
\hline Parameter & $\mathrm{BMI} \leq 25$ & $\mathrm{BMI}>25$ & $P$ value \\
\hline & & $L D-I M R$ & \\
\hline $\mathrm{SD}(\mathrm{HU})$ & $10.2 \pm 0.9$ & $10.2 \pm 1.6$ & $>0.05$ \\
\hline SNR lesions & $4.1 \pm 1.3$ & $5.0 \pm 2.1$ & $>0.05$ \\
\hline \multirow[t]{2}{*}{ Image quality overall score } & $4(0)$ & $4(0)$ & - \\
\hline & & $S L D-I M R$ & \\
\hline $\mathrm{SD}(\mathrm{HU})$ & $14.9 \pm 1.7$ & $16.2 \pm 1.6$ & $>0.05$ \\
\hline SNR lesions & $3.3 \pm 1.1$ & $3.5 \pm 1.2$ & $>0.05$ \\
\hline \multirow[t]{2}{*}{ Image quality overall score } & $4(0)$ & $4(0)$ & $>0.05$ \\
\hline & & $U L D-I M R$ & \\
\hline $\mathrm{SD}(\mathrm{HU})$ & $17.3 \pm 2.2$ & $23.1 \pm 9.0$ & $<0.001^{\mathrm{a}}$ \\
\hline SNR lesions & $2.9 \pm 0.9$ & $3.1 \pm 0.9$ & $>0.05$ \\
\hline Image quality overall score & $4(1)$ & $3(0)$ & $<0.001^{\mathrm{b}}$ \\
\hline
\end{tabular}

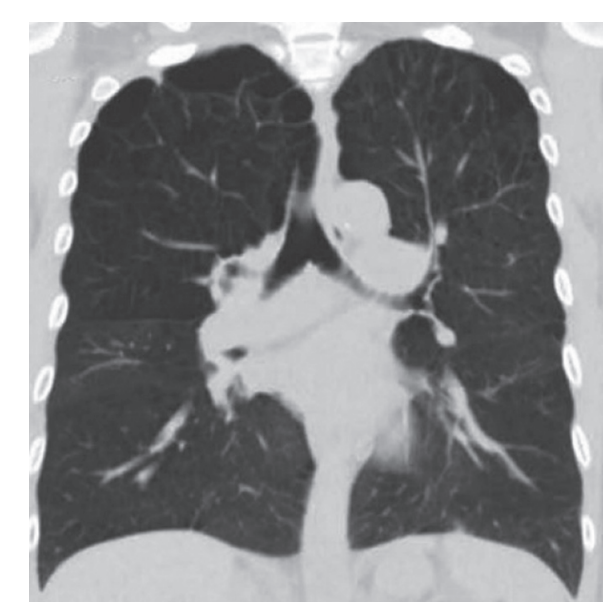

(a)

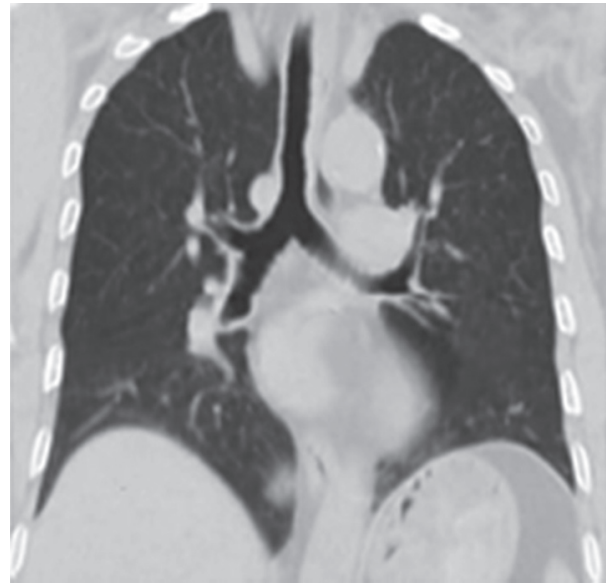

(b)

Figure 2: Comparison of CT values of the aorta between different doses and different algorithms.

TABLE 4: Overall subjective evaluation results.

\begin{tabular}{lccc}
\hline Overall image subjective evaluation score & FBP & HIR & IMR \\
\hline & \multicolumn{3}{c}{ LD-CT } \\
4 & 49 & 50 & 50 \\
3 & 3 & 2 & 2 \\
2 & 0 & 0 & 0 \\
1 & 0 & 0 & 0 \\
$t$ value & 324.7 & 402.5 & 402.5 \\
\hline & \multicolumn{3}{c}{$S L D-C T$} \\
4 & 9 & 9 & 47 \\
3 & 37 & 40 & 5 \\
2 & 6 & 3 & 0 \\
1 & 0 & 0 & 0 \\
$t$ value & 118.7 & 168.96 & 269.1 \\
\hline & \multicolumn{3}{c}{$U L D-C T$} \\
4 & 0 & 0 & 45 \\
3 & 3 & 6 & 7 \\
2 & 36 & 37 & 0 \\
1 & 13 & 9 & 0 \\
$t$ value & 84.4 & 118.7 & 220.9 \\
\hline
\end{tabular}

display evaluation had higher scores. This study found that even though the full model iterative reorganization image has the disadvantage of blocky pixelated performance, it does not affect the normal structure and the observation ability of the lesion. Previous studies have focused on the overall image quality and the display ability of the normal and fine anatomical structures of the lungs, and the evaluation of the display ability of the morphological characteristics of specific lung diseases is still lacking. The results of this study showed that the full-model iterative recombination algorithm and the low-dose-partial iteration algorithm were similar in assessing lung lesions, and both were able to better characterize the periphery of the lungel and can better display lung characteristics such as calcification, necrosis, leaf, speculation, pleural indentation, vacuoles, and ground glass. CT image results showed no significant difference between the subjective image quality scores of the low-doseiterative model and the low-dose full-model iterative recombination algorithm. In the filtered back projection, the ability to significantly decrease calcification evaluation, false positive rate was $14 \%$ higher, more consideration is ignored when the focus is too noisy. There are many granular appearances in CT images, which can be related to the size of the measured voxels, among other reasons. The actual size of the device is related to other reasons. Detection of the lesion size: a measurement result of each group had no significant difference, consistent with the results of other studies [5]; the minimum diameter of lung cancer lesions measured by each algorithm was $1.09 \mathrm{~cm}$ and the 


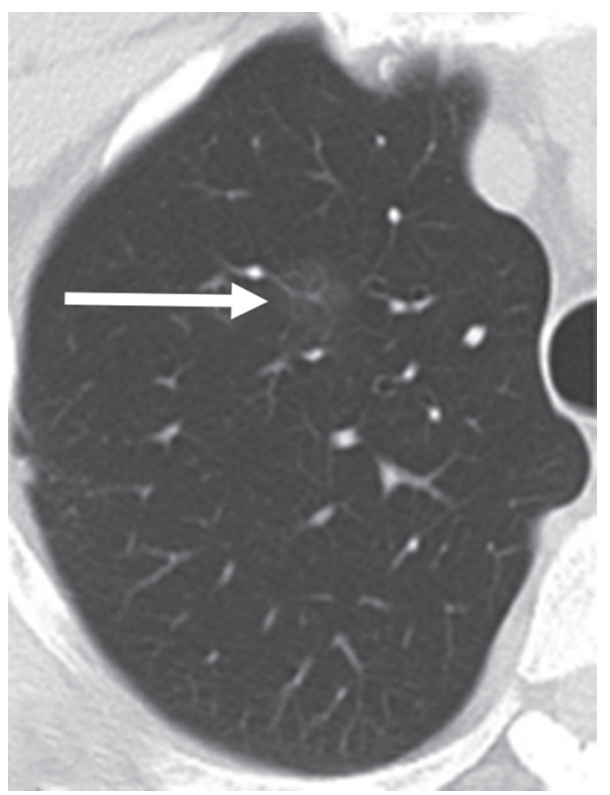

(a)

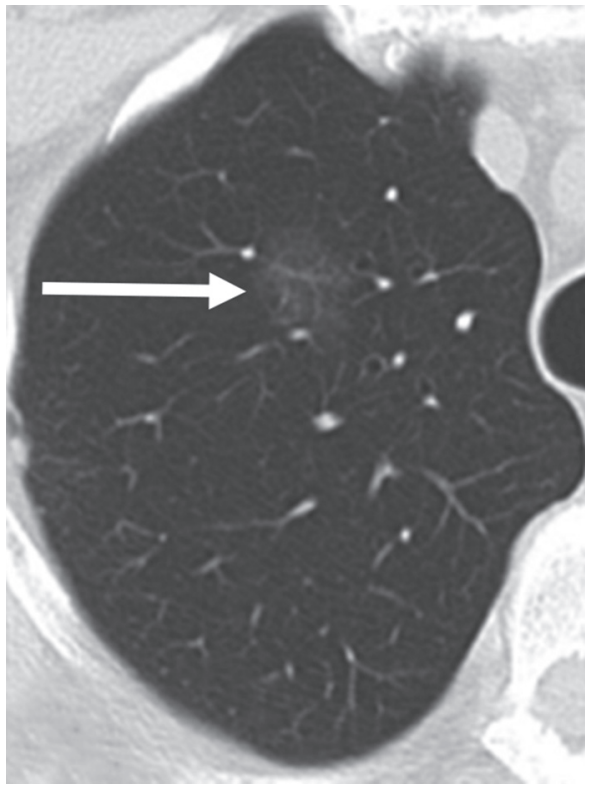

(b)

FIgURE 3: CT image of the lung of case 1.

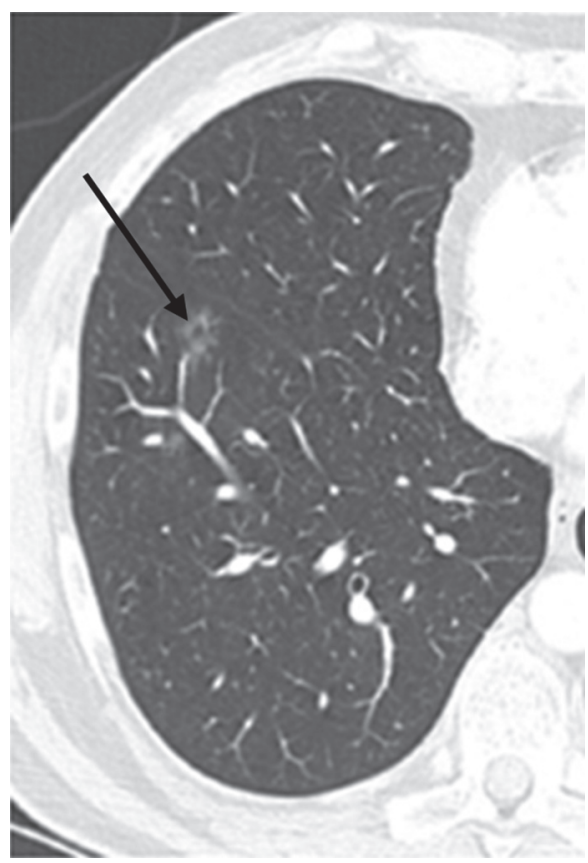

(a)

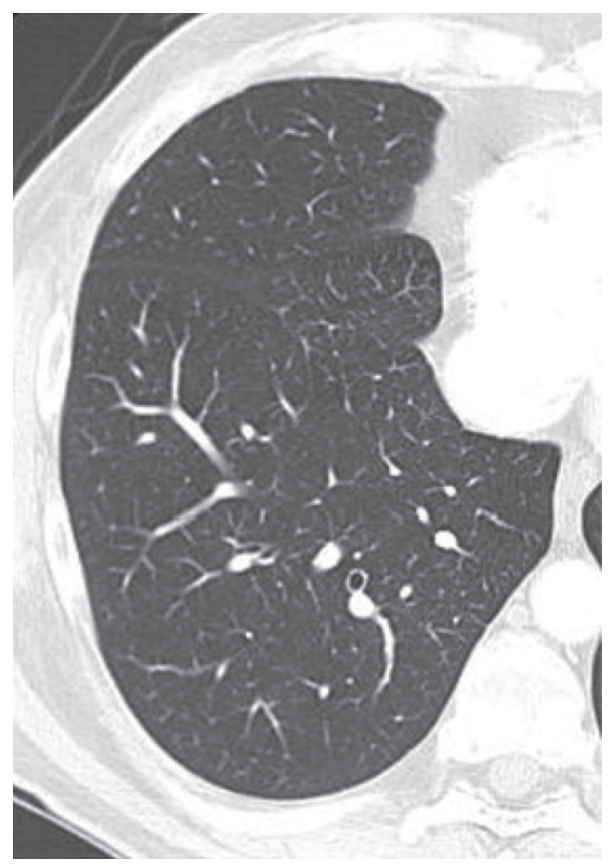

(b)

Figure 4: CT image of the lung of case 2.

maximum diameter was $4.55 \mathrm{~cm}$ for all patients, and it was experimentally confirmed that different algorithms and different dose levels of CT scans did not affect the measurement of lesion size in lung cancer patients. In terms of diagnostic accuracy, the CT diagnostic accuracy under the full-model iterative algorithm was as high as $94 \%$, with an error rate of $6 \%$, which may be caused by the change or disappearance of imaging features after chemotherapy and radiotherapy for lung cancer [6].

There are studies. It is found that the doctor's confidence in diagnosing the lesion is better in patients with high BMI than in patients with lower BMI, which may be related to the nonlinear performance of the algorithm. According to the guidelines for diagnosis and treatment of primary lung cancer in China, chest 


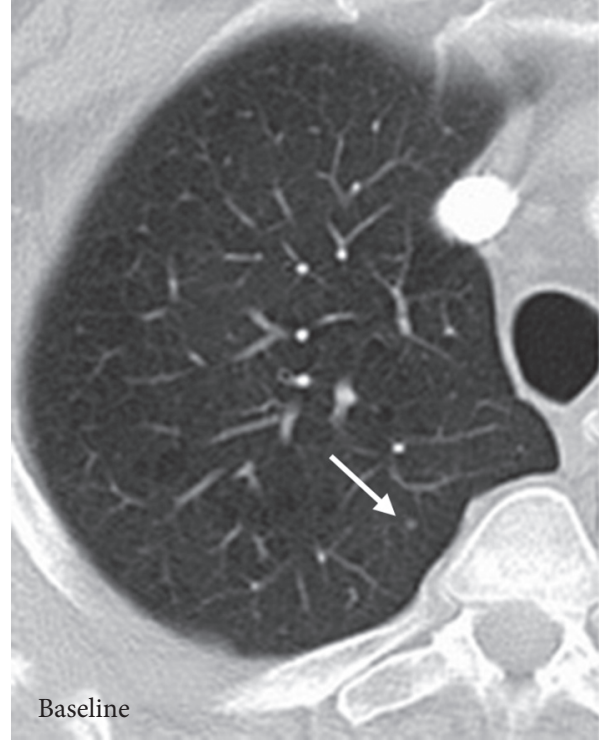

(a)

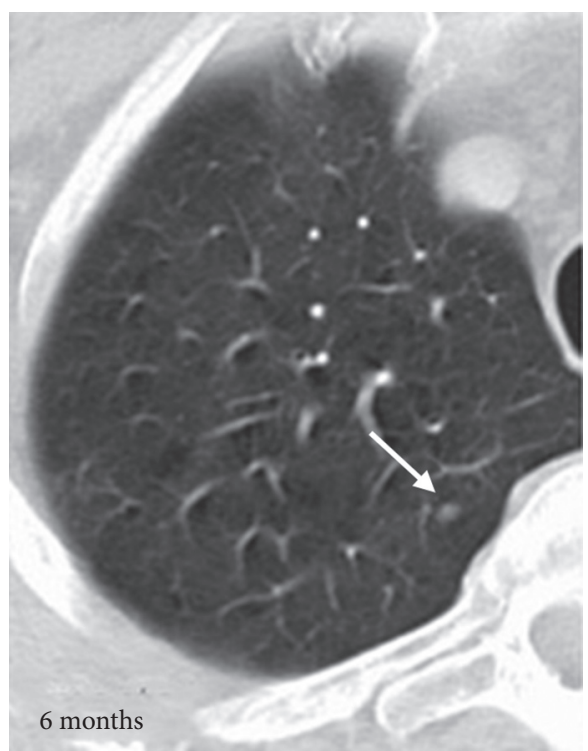

(b)

Figure 5: CT image of the lung in case 3.

CT scan is an important method for the diagnosis, efficacy monitoring, and prognostic evaluation of lung cancer. The ULD-CT combined with IMR technology described in this study can solve the two contradictions between image quality and radiation dose; with the improvement of hardware facilities and computer speed, the reorganization time of the IMR algorithm can be within 3 minutes, and a true green CT scan can be achieved [7].

\section{Conclusion}

The shortcomings of this study: (1) the sample size is relatively small, and the number of lung cancers containing ground glass components is relatively small; (2) the proportion of large BMI is small, which may cause biased results; (3) low dose has not been evaluated in CT enhanced scan; (4) we did not analyze other lesions in the lungs, except lung cancer lesions. In summary, at $80 \mathrm{kVp}$ tube voltage, the ULD-CT based on the IMR algorithm with an ED of $0.3 \mathrm{mSv}$ can still display the imaging characteristics of peripheral lung cancer.

\section{Data Availability}

The data used to support the findings of this study are available from the corresponding author upon request.

\section{Conflicts of Interest}

The authors declare no conflicts of interest.

\section{Authors' Contributions}

Xia Li and Zhanqiang Song contributed equally to this work.

\section{References}

[1] J. Yue, T. Xu, T. Pan et al., "The predictive value of serial $18 \mathrm{f}-\mathrm{fdg}$ pet/CT for recurrence and survival of locally advanced nonsmall cell lung cancer after concurrent chemoradiation therapy," International Journal of Radiation Oncology, Biology, Physics, vol. 93, no. 3, pp. E430-E431, 2015.

[2] $\mathrm{S}$. Wu, "A traffic motion object extraction algorithm," International Journal of Bifurcation and Chaos, vol. 25, no. 14, Article ID 1540039, 2015.

[3] M.-C. Desseroit, D. Visvikis, F. Tixier et al., "Development of a nomogram combining clinical staging with 18F-FDG PET/CT image features in non-small-cell lung cancer stage I-III," European Journal of Nuclear Medicine and Molecular Imaging, vol. 43, no. 8, pp. 1477-1485, 2016.

[4] N. Pécuchet, E. Zonta, A. Didelot et al., "Base-position error rate analysis of next-generation sequencing applied to circulating tumor dna in non-small cell lung cancer: a prospective study," PLoS Medicine, vol. 13, no. 12, Article ID e1002199, 2016.

[5] $\mathrm{S}$. $\mathrm{Wu}$, "Internet public information text data mining and intelligence influence analysis for user intent understanding," Journal of Intelligent and Fuzzy Systems, vol. 38, pp. 487-494, 2020.

[6] S. Datong, F. Lei, J. Yingjian, and Y. Wang, "Effect of scanning and reconstruction parameters on threedimensional volume and ct value measurement of pulmonary nodules: A phantom study," Zhongguo fei ai za zhi = Chinese journal of lung cancer, vol. 20, no. 8, p. 562, 2017.

[7] S. Wu, J. Liu, and L. Liu, "Modeling method of internet public information data mining based on probabilistic topic model," The Journal of Supercomputing, vol. 75, no. 9, pp. 5882-5897, 2019. 